\title{
Stability of smectic phases in hard-rod mixtures
}

\author{
Yuri Martínez-Ratón ${ }^{\text {a) }}$ \\ Grupo Interdisciplinar de Sistemas Complejos (GISC), Departamento de Matemáticas, \\ Escuela Politécnica Superior, Universidad Carlos III de Madrid, Avenida de la Universidad 30, E-28911 \\ Leganés, Madrid, Spain
}

Enrique Velasco ${ }^{\mathrm{b})}$

Departamento de Física Teórica de la Materia Condensada and Instituto de Ciencia de Materiales Nicolás Cabrera, Universidad Autónoma de Madrid, E-28049 Madrid, Spain

Luis Mederos ${ }^{\mathrm{c}}$

Instituto de Ciencia de Materiales, Consejo Superior de Investigaciones Científicas, E-28049 Cantoblanco, Madrid, Spain

(Received 13 June 2005; accepted 11 July 2005; published online 14 September 2005)

Using density-functional theory, we have analyzed the phase behavior of binary mixtures of hard rods of different lengths and diameters. Previous studies have shown a strong tendency of smectic phases of these mixtures to segregate and, in some circumstances, to form microsegregated phases. Our focus in the present work is on the formation of columnar phases which some studies, under some approximations, have shown to become thermodynamically stable prior to crystallization. Specifically we focus on the relative stability between smectic and columnar phases, a question not fully addressed in previous work. Our analysis is based on two complementary perspectives: on the one hand, an extended Onsager theory, which includes the full orientational degrees of freedom but with spatial and orientational correlations being treated in an approximate manner; on the other hand, we formulate a Zwanzig approximation of fundamental-measure theory on hard parallelepipeds, whereby orientations are restricted to be only along three mutually orthogonal axes, but correlations are faithfully represented. In the latter case novel, complete phase diagrams containing regions of stability of liquid-crystalline phases are calculated. Our findings indicate that the restricted-orientation approximation enhances the stability of columnar phases so as to preempt smectic order completely while, in the framework of the extended Onsager model, with full orientational degrees of freedom taken into account, columnar phases may preempt a large region of smectic stability in some mixtures, but some smectic order still persists. () 2005 American Institute of Physics. [DOI: 10.1063/1.2013251]

\section{INTRODUCTION}

Recent work has shown that strong segregation effects arise in smectic $(S)$ phases of hard rods of equal diameter but different lengths when the two components are mixed in varying proportions. In seminal work Koda and Kimura ${ }^{1}$ analyzed a binary mixture of parallel hard cylinders using Onsager approximation, and applied a stability analysis. They found two types of $S$ phases: one in which layers are identical and contain a mixture of both types of particles, and another, microsegregated phase, which consists of alternating layers of different compositions. More recent work has focused on more general mixtures. For example, van Roij and Mulder $^{2}$ studied a mixture of parallel cylinders of different diameters using Onsager theory and a more complete bifurcation analysis. They observed that, for length ratios higher than 1:5, N-N segregation (where $N$ stands for nematic phase) preempts the $N-S$ and $N-C$ phase transitions ( $C$ stands

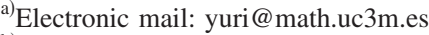

b) Electronic mail: enrique.velasco@uam.es

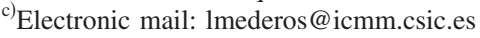

for columnar phase). More recent work by Cinacchi et al. ${ }^{3,4}$ used an extended Onsager theory (EOT) on systems of hard spherocylinders (HSPCs), but lifting the restriction on orientations, and complete phase diagrams were obtained containing $I$ (isotropic), $N$, and $S$, phases. As was expected, a strong tendency toward segregation was observed, and microsegregated phases did also arise in the model, consisting of layers made up of the component in larger proportion-the shorter rods-alternating with weakly populated and highly interpenetrated layers of the longer rods.

In the work by Cinacchi $e t$ al. the only spatially ordered phase considered was the $S$ phase. $C$ phases are very common in nature, even in systems composed of prolate molecules. A simple, one-component model is known not to exhibit $C$ ordering, which is preempted by the smectic phase. However, the $C$ phase can be stabilized prior to crystallization in mixtures of HSPC particles with orientations restricted to be parallel, as shown by Stroobants et al. ${ }^{5}$ using computer simulation. Bohle et al., ${ }^{6}$ using a density functional based on the weighted-density approximation, considered the effect of polydispersity on the stabilization of ordered phases in systems of parallel hard rods. Their conclusion is similar 
to that obtained previously by Bates and Frenkel, ${ }^{7}$ viz., increasing the degree of polydispersity stabilizes the $C$ phase with respect to the $S$ phase. An identical behavior was observed in mixtures with perfectly bimodal length distributions ${ }^{6}$ (i.e., two-component mixtures).

It is intuitively clear that orientational fluctuations, not taken into account in all of these studies, will tend to destroy columnar order, but the extent to which this statement is true in mixtures of hard rods is still unknown. As a consequence, a general concern is the question of whether phases of lower symmetry, $C$ and crystal, may preempt all the structures observed in preliminary studies; in particular, all previous studies have shown the $C$ phase to be stable for systems of perfectly parallel rods, preempting the occurrence of the smectic phase for sufficiently large length ratios. Also, in connection with the work by Cinacchi et al., ${ }^{3}$ a number of questions remain unanswered, particularly in relation with the theoretical model (EOT), its particular implementation, and the approximations used (parametrization of the distribution functions, etc.). These are the aims of the present paper. In this paper we have not considered the occurrence of crystal phases, largely for two reasons: one is that in mixtures the crystal phase will certainly tend to appear at relatively high packing fractions, due to packing constraints, which will leave a large region where other spatially ordered phases may appear. Another reason is that the EOT is not well suited to study crystallization. Therefore we will limit ourselves to studying the instability against general spatial fluctuations of the $N$ phase.

In the present paper we address the above questions from two perspectives. One is the calculation, within EOT, of the line where the nematic phase of the mixture gets unstable with respect to columnar-phase fluctuations. This line sets an approximate upper limit for the region where smectic phases should be stable. The other is the numerical minimization of a fundamental-measure functional as applied to systems of hard rods of square transversal section [hard parallelepipeds (HPs)] in the Zwanzig approximation, i.e., considering that the rods can assume only three possible orientations, namely, along the Cartesian axes $x y z$. In this way, by using a completely different approach which possesses some advantages (better treatment of hard-core correlations and exact formulation of excluded-volume effects) and some disadvantages (restricted orientations), we may verify whether the predictions of Onsager theory are artifacts of the theory or of its particular implementation. We will see that some of the predictions of the two models agree, which gives us some confidence as to the realistic nature of the results. On the other hand, the results from the fundamental-measure theory (FMT) predict new structures, not observed in the Onsager theory, which poses interesting new questions and opens up new avenues of research-of course the extent to which these structures are artifacts of the Zwanzig approximation or peculiarities of the particle's shape is as yet unknown.

In Sec. II we briefly remind the reader the ingredients of the two theoretical approaches. It is also devoted to present the methodology used to locate the spinodal line for $C$ ordering in the context of the above density-functional theories. In Sec. III we present a few results pertaining to the EOT used previously ${ }^{3}$ to analyze phase behavior in HSPC mixtures, summarizing relevant features that appear in the phase diagrams of a few selected mixtures. Complete phase diagrams, containing $I, N$, and $S$ phases, are presented. Our purpose is to compare the corresponding results with those obtained using the Zwanzig approximation of FMT as applied to HP particles and for equivalent mixtures. Density and orderparameter profiles are shown to better demonstrate the structure of the spatially ordered $S$ phases. Also, spinodal lines signaling the instability against $C$-type fluctuations are shown. The location of this line in connection with the stability of the $S$ phases is discussed. We end in Sec. IV with a summary of the results and some final conclusions drawn from the comparison of the results obtained with the two theoretical approaches.

\section{THEORETICAL APPROACHES}

In this section we use two versions of the densityfunctional theory, a modified Onsager theory and fundamental-measure theory in the Zwanzig approximation, to obtain phase diagrams of a few selected mixtures of hard rods. Attention is restricted to spatially disordered phases [isotropic $(I)$ and nematic $(N)$ ] and also to smectic $(S)$ phases. The more ordered columnar $(C)$ phase will be considered in Sec. II C.

\section{A. Extended Onsager theory for mixtures of hard spherocylinders}

We analyze the phase behavior of binary mixtures of hard spherocylinders (HSPs) of length $L_{s}(s=1,2)$ and the same diameter $\sigma$. The one-particle distribution functions of each component will be denoted by $\rho_{s}(\mathbf{r}, \hat{\mathbf{\Omega}})$. The density functional for the Helmholtz free energy $\mathcal{F}$ is written, as usual, as a sum of ideal $\mathcal{F}_{\text {id }}$ and excess $\mathcal{F}_{\text {ex }}$ terms:

$$
\mathcal{F}\left[\left\{\rho_{s}\right\}\right]=\mathcal{F}_{\text {id }}\left[\left\{\rho_{s}\right\}\right]+\mathcal{F}_{\text {ex }}\left[\left\{\rho_{s}\right\}\right],
$$

with the ideal part given by

$$
\beta \mathcal{F}_{\mathrm{id}}\left[\left\{\boldsymbol{\rho}_{s}\right\}\right]=\sum_{s=1}^{2} \iint d \mathbf{r} d \hat{\mathbf{\Omega}} \rho_{s}(\mathbf{r}, \hat{\mathbf{\Omega}})\left\{\ln \rho_{s}(\mathbf{r}, \hat{\mathbf{\Omega}})-1\right\},
$$

where $\beta=1 / k T$. The excess part is given by

$$
\beta \mathcal{F}_{\text {ex }}\left[\left\{\rho_{s}\right\}\right]=\int_{V} d \mathbf{r} \Phi_{\text {ex }}\left(\mathbf{r} ;\left\{\rho_{s}\right\}\right),
$$

where the excess free-energy density per unit thermal energy, $\Phi_{\mathrm{ex}}\left(\mathbf{r} ;\left\{\rho_{s}\right\}\right)$, is written, in the EOT, as

$$
\begin{aligned}
\Phi_{\mathrm{ex}}\left(\mathbf{r} ;\left\{\boldsymbol{\rho}_{s}\right\}\right)= & \Psi(\eta) \sum_{s=1}^{2} \sum_{t=1}^{2} \int_{V} d \mathbf{r}^{\prime} \iint d \hat{\mathbf{\Omega}}^{\prime} \hat{\mathbf{\Omega}}^{\prime} \boldsymbol{\rho}_{s}(\mathbf{r}, \hat{\mathbf{\Omega}}) \\
& \times \boldsymbol{\rho}_{t}\left(\mathbf{r}^{\prime}, \hat{\mathbf{\Omega}}^{\prime}\right) f_{s t}\left(\mathbf{r}-\mathbf{r}^{\prime}, \hat{\mathbf{\Omega}}, \hat{\mathbf{\Omega}}^{\prime}\right) .
\end{aligned}
$$

$f_{s t}$ are overlap functions for the three different interactions (unity if particles overlap and zero otherwise), and $\Psi(\eta)$ is a prefactor that depends on the mean packing fraction $\eta$ and that is chosen to make the theory recover the second virial 
coefficient exactly and approximate the remaining virial coefficients in terms of the second coefficient, in the manner proposed by Parsons ${ }^{8}$ and Lee. ${ }^{9}$ Our implementation of the present theory (see details $\mathrm{in}^{3,4}$ ) involves first using the decoupling approximation,

$$
\rho_{s}(\mathbf{r}, \hat{\mathbf{\Omega}}) \approx \rho_{s}(\mathbf{r}) h_{s}(\hat{\mathbf{\Omega}})
$$

(i.e., particle positions and orientations, characterized, respectively, by the distribution functions $\rho_{s}$ and $h_{s}$, are assumed to be decoupled at the level of the one-particle distribution functions), and then parametrizing the two distribution functions in terms of suitably normalized exponential functions:

$$
\begin{aligned}
\rho_{s}(\mathbf{r}, \hat{\mathbf{\Omega}}) \approx & \rho_{s}(\mathbf{r}) h_{s}(\hat{\mathbf{\Omega}})=x_{s} \rho\left[\frac{e^{\lambda_{s} \cos q z}}{I_{0}\left(\lambda_{s}\right)}\right] \\
& \times\left[\frac{e^{\Lambda_{s} P_{2}(\cos \theta)}}{2 \pi \int_{-1}^{1} d x e^{\Lambda_{s} P_{2}(x)}}\right],
\end{aligned}
$$

where $q=2 \pi / d$ is the wave number of the smectic undulation with period $d, \Lambda_{s}$ are variational parameters describing the orientational order $\left(\Lambda_{s}=0\right.$ in the $I$ phase and $\Lambda_{s} \neq 0$ in the $N$ and $S$ phases), $\left\{\lambda_{s}\right\}$ are variational parameters describing the positional order ( $\lambda_{s}=0$ in the $N$ phase), $x_{s}$ the molar fractions of species $s, \rho$ the mean density, and $I_{0}(x)$ is the modified Bessel function of order 0. Equation (1) should be minimized with respect to the variational parameters $\left\{\lambda_{s}\right\},\left\{\Lambda_{s}\right\}$, and $d$ to find the equilibrium density profiles and the free energy for particular values of $\rho$ and $x$. The Gibbs free energy per particle $g$ is obtained from

$$
g=\frac{F}{N}+\frac{P}{\rho}, \quad P=\left(\rho \frac{\partial}{\partial \rho}-1\right) \frac{F}{V},
$$

where $P$ is the pressure and $F$ is the equilibrium Helmholtz free energy or directly from an appropriate derivate of the free-energy density functional evaluated at the equilibrium profiles. Applying a Maxwell double-tangent construction on $g(x)$ for fixed $P$ allows us to find the coexistence values of the different phases in the usual way and to obtain the phase diagrams in the pressure-composition plane.

\section{B. Fundamental-measure theory for mixtures of hard parallelepipeds in the Zwanzig approximation}

The FMT used here has been described in detail in Ref. 10 , so that only a brief sketch is provided here in order to show how it can be numerically implemented to calculate phase diagrams of mixtures. We consider a system of HPs, i.e., hard rods of rectangular shape. We continue to use the notation introduced in Sec. II A for the dimensions of the particles; in this case we denote by $L_{s}$ the length of a parallelepiped of species $s$, with $\sigma_{s}$ being the breadth of the particles (assumed to have a square section).

We have used the fundamental-measure functional for HP in the approximation that orientations are restricted to lie along three mutually orthogonal directions (Zwanzig approximation), taken to be the $x y z$ axes. Since the unit vector $\hat{\mathbf{\Omega}}$ only has three possible orientations, one can think of the one-particle distribution functions $\rho_{s}(\mathbf{r}, \hat{\mathbf{\Omega}})$ as being expressed in terms of a set of Dirac-delta functions along the three axes:

$$
\rho_{s}(\mathbf{r}, \hat{\mathbf{\Omega}})=\sum_{\mu} \rho_{s \mu}(\mathbf{r}) \delta\left(\hat{\mathbf{\Omega}}-\hat{\mathbf{e}}_{\mu}\right),
$$

where $\hat{\mathbf{e}}_{\mu}, \mu=1,2,3$, are unit vectors along the three perpendicular directions $x y z$, respectively. Note that this expression does not assume a decoupling approximation since it implies a strong mixing of spatial and orientational degrees of freedom. The coefficients of these expansions, $\rho_{s \mu}(\mathbf{r})$, are the local density of species $s$ parallel to the $\mu$-axe and represent quantitatively the orientational order in the system in the Zwanzig approximation. The free-energy functional will be given by Eqs. (1)-(3) and (8), where the excess part of the free-energy density in reduced thermal units, obtained in Ref. 10 , has the form

$$
\Phi_{\mathrm{ex}}\left(\mathbf{r} ;\left\{\rho_{s}\right\}\right)=-n_{0} \ln \left(1-n_{3}\right)+\frac{\mathbf{n}_{1} \cdot \mathbf{n}_{2}}{1-n_{3}}+\frac{n_{2 x} n_{2 y} n_{2 z}}{\left(1-n_{3}\right)^{2}},
$$

with the functions $\left\{n_{\alpha}\right\}(\alpha=\{0,1 x, 1 y, 1 z, 2 x, 2 y, 2 z, 3\})$ being weighted densities obtained as

$$
n_{\alpha}(\mathbf{r})=\sum_{s=1}^{2} \sum_{\mu=1}^{3} \int_{V} d \mathbf{r}^{\prime} \rho_{s \mu}\left(\mathbf{r}^{\prime}\right) \omega_{s \mu}^{(\alpha)}\left(\mathbf{r}-\mathbf{r}^{\prime}\right),
$$

where $\omega_{s \mu}^{(\alpha)}$ are characteristic functions whose spatial integrals give the fundamental measures of the particles (edge length, surface, and volume). In this case, instead of the parametrization Eq. (6), used in the EOT, we use a Fourier expansion to represent smectic order:

$$
\rho_{s \mu}(\mathbf{r})=x_{s} \rho\left[\sum_{k=0}^{K} \alpha_{s \mu}^{(k)} \cos q k z\right] \gamma_{s \mu},
$$

(this expression is obviously also valid for the $N$ phase), where $\left\{\alpha_{s \mu}^{(k)}\right\}$ are Fourier amplitudes (we impose $\alpha_{s \mu}^{(0)}=1$ and uniaxial symmetry, reflected in the condition $\alpha_{s x}^{(k)}=\alpha_{s y}(k)$. The coefficients $\left\{\gamma_{s \mu}\right\}$ (which play the role of $f_{s}(\hat{\boldsymbol{\Omega}})$ in the Onsager theory) are the spatially average probabilities to find a particle of species $s$ parallel to the $\mu$ axis (again uniaxial symmetry implies $\gamma_{s x}=\gamma_{s y}$ ). The number of terms in the expansion, $K+1$, is chosen so that $\alpha_{s \mu}^{(K)}<10^{-7}$.

In the nematic limit $\rho_{s}(\mathbf{r}, \hat{\mathbf{\Omega}})=\rho_{s} h_{s}(\hat{\mathbf{\Omega}})$, and the coefficients $\gamma_{s \mu}$ are easily obtained in terms of the nematic order parameters $\left\{Q_{s}\right\}$ (with $-1 / 2 \leqslant Q_{s} \leqslant 1$ ) by imposing the conditions

$$
1=\int d \hat{\mathbf{\Omega}} h_{s}(\hat{\mathbf{\Omega}}), \quad Q_{s}=\int d \hat{\mathbf{\Omega}} h_{s}(\hat{\mathbf{\Omega}}) P_{2}\left(\hat{\mathbf{\Omega}} \cdot \mathbf{e}_{z}\right),
$$

$\left[P_{2}(x)\right.$ is the second-order Legendre polynomial] which lead to

$$
\gamma_{s \mu}=\frac{1}{3}\left[1+\left(3 \delta_{\mu z}-1\right) Q_{s}\right],
$$

with $\delta_{\mu \nu}$ the Kronecker delta. We have selected the nematic director to be parallel to the $z$ axis.

With these definitions the weighted densities can be easily calculated from Eqs. (10) and (11) as 


$$
n_{\alpha}(z)=\rho \sum_{s \mu} x_{s} \gamma_{s \mu} \sum_{k=0}^{n} \alpha_{s \mu}^{(k)} \hat{\omega}_{s \mu}^{(\alpha)}(q k) \cos (q k z)
$$

where the functions $\hat{\omega}_{s \mu}^{(\alpha)}$ are the Fourier transforms of effective one-dimensional weights resulting from partial integration over $x$ and $y$ :

$$
\begin{aligned}
& \hat{\omega}_{s \mu}^{(0)}(q k)=j_{0}\left(\frac{q}{2} k \sigma_{\mu z}^{s}\right), \\
& \hat{\omega}_{s \mu}^{(1 \nu)}(q k)=\sigma_{\mu \nu}^{s} j_{\delta_{\nu z}}\left(\frac{q}{2} k \sigma_{\mu z}^{s}\right), \\
& \hat{\omega}_{s \mu}^{(2 \nu)}(q k)=\frac{v_{s}}{\sigma_{\mu \nu}^{s}} j_{1-\delta_{\nu z}}\left(\frac{q}{2} k \sigma_{\mu z}^{s}\right), \\
& \hat{\omega}_{s \mu}^{(3)}(q k)=v_{s} j_{1}\left(\frac{q}{2} k \sigma_{\mu z}^{s}\right),
\end{aligned}
$$

with $v_{s}=L_{s} \sigma_{s}^{2}$ the particle volumes, $\sigma_{\mu \nu}^{s}=\sigma_{s}+\left(L_{s}-\sigma_{s}\right) \delta_{\mu \nu}$, $j_{0}(x)=\cos x$, and $j_{1}(x)=\sin x / x$.

The ideal part of the free-energy density in reduced thermal units for this model is

$$
\Phi_{\mathrm{id}}(z)=\sum_{s \mu} \rho_{s \mu}(z)\left[\ln \rho_{s \mu}(z)-1\right]
$$

Thus the total free energy per unit volume can be calculated as

$$
\frac{\beta \mathcal{F}}{V}=\frac{1}{d} \int_{0}^{d} d z\left[\Phi_{\mathrm{id}}(z)+\Phi_{\mathrm{ex}}(z)\right]
$$

Now Eq. (17) is minimized with respect to the parameters $\gamma_{s \mu}, \alpha_{s \mu}^{(k)}$, and $d$ to find the equilibrium density profiles for fixed $\rho$ and $x$. The pressure can be evaluated from

$$
\begin{aligned}
\beta P= & \frac{1}{d} \int_{0}^{d} d z\left\{\frac{n_{0}(z)}{1-n_{3}(z)}+\frac{\mathbf{n}_{1}(z) \cdot \mathbf{n}_{2}(z)}{\left[1-n_{3}(z)\right]^{2}}\right. \\
& \left.+2 \frac{n_{2 x}(z) n_{2 y}(z) n_{2 z}(z)}{\left[1-n_{3}(z)\right]^{3}}\right\},
\end{aligned}
$$

and the phase diagram is obtained as indicated in Sec. II A. The results for phase diagrams of different mixtures (including the one-component limit) pertaining to this theory, and also to the EOT approach outlined in Sec. II B, will be presented in Sec. III. Also, density and order-parameter profiles for the smectic phases will be shown in order to better grasp the structure of these phases. These profiles are defined as follows. The total density profile is

$$
\rho_{s}(z)=\sum_{\mu} \rho_{s \mu}(z)
$$

while the nematic order-parameter profile is defined by

$$
Q_{s}(z)=\frac{1}{2}\left[\frac{3 \rho_{s z}(z)}{\rho_{s}(z)}-1\right] .
$$

\section{Instability against spatial fluctuations}

In this section we present the formalism used to analyze the instabilities against spatial fluctuations. We first describe the bifurcation analysis that we have used to locate the instability points of the $N$ phase against spatial ordering. Our aim is to see whether the phase behavior of the above HSPC mixtures is strongly modified by the presence of spatially ordered phases with a symmetry lower than that of the $S$ phase. In the present paper we are interested in locating the spinodal line corresponding to $C$ ordering, since this is the phase that, following previous work on parallel hard rods, might become stable prior to crystallization.

Instabilities against both $S$ and $C$ fluctuations can be explored at the same time by examining the response function of the $N$ phase against general spatial fluctuations of a wave vector $\mathbf{q}$. The free-energy change $\delta F$ associated with fluctuations $\delta \rho_{s}$ in the one-particle distribution functions of the two components of the mixture can be expressed, up to second order in the fluctuations, in terms of the second functional derivative of the free-energy functional evaluated at the nematic phase,

$$
\begin{aligned}
\delta F= & \frac{1}{2} \sum_{s=1}^{2} \sum_{t=1}^{2} \int_{V} d \mathbf{r} \int d \hat{\mathbf{\Omega}} \int_{V} d \mathbf{r}^{\prime} \int d \hat{\mathbf{\Omega}}^{\prime} \\
& \times\left.\frac{\delta^{2} \mathcal{F}}{\delta \rho_{s}(\mathbf{r}, \hat{\mathbf{\Omega}}) \delta \rho_{t}\left(\mathbf{r}^{\prime}, \hat{\mathbf{\Omega}}^{\prime}\right)}\right|_{\mathbf{N}} \delta \rho_{s}(\mathbf{r}, \hat{\mathbf{\Omega}}) \delta \rho_{t}\left(\mathbf{r}^{\prime}, \hat{\mathbf{\Omega}}^{\prime}\right) .
\end{aligned}
$$

The instability is signaled by the equation $\delta F=0$. Two different approaches can be followed here. In one, we assume that fluctuations are realized at constant mean density $\rho$, so that the minimization of $\mathcal{F}$ is performed via a Lagrange multiplier or, equivalently, by choosing fluctuations $\delta \rho_{s}$ that conserve the mean density. This is the approach to be followed in the canonical ensemble where the composition $x$ and the mean density $\rho$ are fixed. In the other approach, general fluctuations, not necessarily mean density conserving, are considered, and the second functional derivative is most conveniently expressed in terms of the direct correlation function $C_{s t}$ evaluated at the nematic phase,

$$
\begin{aligned}
\left.\frac{\delta^{2}[\beta \mathcal{F}]}{\delta \rho_{s}(\mathbf{r}, \hat{\mathbf{\Omega}}) \delta \rho_{t}\left(\mathbf{r}^{\prime}, \hat{\mathbf{\Omega}}^{\prime}\right)}\right|_{\mathrm{N}}= & \frac{\delta\left(\mathbf{r}-\mathbf{r}^{\prime}\right) \delta\left(\hat{\mathbf{\Omega}}-\hat{\mathbf{\Omega}}^{\prime}\right) \delta_{s t}}{\rho_{s}^{(N)}(\hat{\boldsymbol{\Omega}})} \\
& -C_{s t}\left(\mathbf{r}-\mathbf{r}^{\prime}, \hat{\mathbf{\Omega}}, \hat{\mathbf{\Omega}}^{\prime}\right),
\end{aligned}
$$

where $C_{s t}$ is the direct correlation function evaluated at the nematic phase, 


$$
C_{s t}\left(\mathbf{r}-\mathbf{r}^{\prime}, \hat{\mathbf{\Omega}}, \hat{\mathbf{\Omega}}^{\prime}\right)=-\left.\frac{\delta^{2}\left[\beta \mathcal{F}_{\mathrm{ex}}\right]}{\delta \rho_{s}(\mathbf{r}, \hat{\mathbf{\Omega}}) \delta \rho_{t}\left(\mathbf{r}^{\prime}, \hat{\mathbf{\Omega}}^{\prime}\right)}\right|_{\rho_{s}(\mathbf{r}, \hat{\mathbf{\Omega}})=\rho_{s}^{(N)}(\hat{\mathbf{\Omega}})} .
$$

This route is followed in the calculations using the isobaric ensemble where the quantities to be fixed are the composition $x$ and the pressure $P$. Depending on the ensemble used the fluctuation has to be chosen accordingly. For the Onsager model we have chosen to use the canonical ensemble; in the spirit of the decoupling approximation, we take

$$
\delta \rho_{s}(\mathbf{r}, \hat{\mathbf{\Omega}})=\epsilon_{s} e^{i \mathbf{q} \cdot \mathbf{r}} \rho_{s}^{(N)}(\hat{\mathbf{\Omega}})=\epsilon_{s} \rho_{s} e^{i \mathbf{q} \cdot \mathbf{r}} h_{s}^{(N)}(\hat{\mathbf{\Omega}}),
$$

where $h_{s}^{(N)}$ is the orientational distribution function of the nematic phase, $\mathbf{q}$ an arbitrary wave vector, and $\epsilon_{s}$ an amplitude giving the strength of the fluctuation. The above fluctuation conserves the value of the order parameters $\left\{Q_{s}\right\}$ as the mean density is fixed. The increase in free energy can be written in matrix form as

$$
\frac{\delta[\beta \mathcal{F}]}{V}=\frac{1}{2} \sum_{s=1}^{2} \sum_{t=1}^{2} \epsilon_{s} \rho_{s} \mathcal{T}_{s t} \epsilon_{t} \rho_{t}=\frac{1}{2} \mathbf{a}^{T} \cdot \mathcal{T} \cdot \mathbf{a},
$$

with $\mathbf{a}^{T}=\left(a_{1}, a_{2}\right)$, and $a_{s}=\epsilon_{s} \rho_{s}$ the components of a twodimensional perturbation vector. The stability of the nematic phase against spatial fluctuations can be assessed by examining the signs of the eigenvectors of the $2 \times 2-\mathcal{T}$ matrix, with

$$
\mathcal{T}_{s t}=\mathcal{T}_{s t}(x ; \mathbf{q}, \rho) \equiv \frac{\delta_{s t}}{\rho_{s}}-\left\langle\left\langle\hat{C}_{s t}\left(\mathbf{q}, \hat{\mathbf{\Omega}}, \hat{\mathbf{\Omega}}^{\prime}\right)\right\rangle\right\rangle_{f_{s}^{(N)}, f_{t}^{(N)}} .
$$

Here $\hat{C}_{s t}$ are Fourier transforms of the direct correlation function. In practice we proceed as follows. Instability is signaled by one of the eigenvalues of $\mathcal{T}$ or, equivalently, the determinant of $\mathcal{T}$, becoming negative. Then, at fixed composition $x$, we look for the value of $\rho$ for which the four equations

$$
\operatorname{det} \mathcal{T}=0, \quad \frac{\partial}{\partial q_{\mu}} \operatorname{det} \mathcal{T}=0, \quad \mu=1,2,3
$$

are satisfied simultaneously $\left(q_{\mu}=q_{x}, q_{y}, q_{z}\right.$ for $\mu=1,2,3$, respectively). In the case of the HSPC model, the $C$ phase consists of columns of particles arranged into a twodimensional triangular lattice perpendicular to the nematic director, and the two-dimensional ( $x y$ plane) perturbing wave that makes the fluid unstable will have a $\mathbf{q}$ vector with nonzero $x$ and $y$ components. An instability associated with wave vectors $\mathbf{q}=(0,0, q)$, i.e., a wave along the $z$ direction (parallel to the nematic director), will signal instability against smectic fluctuations. For the system of HP the underlying lattice of the $C$ phase is square, so that instability will be signaled by wave vectors $\mathbf{q}=(q, 0,0)$ or $(0, q, 0)$.

In the case of the FMT approximation the problem can be cast in matrix form by introducing the fluctuation

$$
\delta \rho_{s}(\mathbf{r}, \hat{\mathbf{\Omega}})=\rho_{s} \sum_{\mu=1}^{3} \epsilon_{s \mu} e^{i \mathbf{q} \cdot \mathbf{r}} \delta\left(\hat{\mathbf{\Omega}}-\hat{\mathbf{e}}_{\mu}\right) .
$$

We remark that in this case the decoupling approximation is not invoked, so the coupling between positional and orienta- tional degrees of freedom, assumed in the implementation of the theory, is maintained. The parameters $\left\{\boldsymbol{\epsilon}_{s \mu}\right\}$ are taken to be arbitrary, as it corresponds to a general fluctuation. In particular, they are all taken to be independent, which is equivalent to saying that the values of the order parameters $\left\{Q_{s}\right\}$ are allowed to vary as the perturbation is applied, in contrast with the method used in the Onsager model. This is done for the sake of convenience, and has no practical importance given that the condition for instability is searched for in the space $(\rho, \mathbf{q})$, and the order parameters depend on the mean density $\rho$. The $\mathcal{T}$ matrix now has six dimensions:

$$
\mathcal{T}_{s \mu, t \nu}=\frac{\delta_{s t} \delta_{\mu \nu}}{\rho_{s \mu}}-\hat{C}_{s \mu, t \nu}(\mathbf{q}),
$$

with

$$
C_{s \mu, t \nu}\left(\mathbf{r}-\mathbf{r}^{\prime}\right)=-\left.\frac{\delta^{2}\left[\beta \mathcal{F}_{\mathrm{ex}}\right]}{\delta \rho_{s \mu}(\mathbf{r}) \delta \rho_{t \nu}\left(\mathbf{r}^{\prime}\right)}\right|_{\rho_{s \mu}(\mathbf{r})=\rho_{s \mu}}
$$

being the relevant direct correlation function and $\hat{C}_{s \mu, t \nu}(\mathbf{q})$ its Fourier transform. Explicit expressions for this function will be presented in the Appendix.

\section{RESULTS}

Although the HSP and HP models are geometrically different (HSP particles have a semispherical cap which is absent in HP particles, and their sections are circular and square, respectively), and their theoretical treatment is quite different, the corresponding phase diagrams will be compared in this section, in the hope that we can extract some useful information about their actual phase behavior. This comparison can be made provided we use comparable aspect ratios $\kappa_{s}$; we should take into account the relation

$$
\kappa_{s}^{\mathrm{HSPC}} \equiv\left(\frac{L_{s}+\sigma}{\sigma}\right)_{\mathrm{HSPC}} \leftrightarrow \kappa_{s}^{\mathrm{HP}} \equiv\left(\frac{L_{s}}{\sigma}\right)_{\mathrm{HP}} .
$$

\section{A. Phase diagrams for pure components}

Before showing the results for mixtures, and in order to have a feeling as to how the two theoretical models can be compared and how definite predictions can be drawn from this comparison, it is convenient to recall the results obtained for the corresponding one-component systems using the same theoretical models that will be used later for mixtures. The phase diagrams for the pure systems are shown in Figs. 1 and 2 both in the packing fraction-aspect ratio plane (we should remind the definitions of the corresponding aspect ratios, $\kappa^{\mathrm{HSPC}}$ and $\kappa^{\mathrm{HP}}$, respectively). A quick comparison of the two phase diagrams indicates that the $I N$ phase transition is qualitatively similar: it is of first order and, for decreasing aspect ratio, ends at some value of $\kappa$, below which the $N$ phase ceases to be stable and is superseded by the spatially ordered $S$ phase or other more ordered phases. For HP particles the phase diagram has been analyzed in some detail using the FMT approach ${ }^{11}$ (see Fig. 2); it is relatively complex, with the $C$ phase becoming stable in a narrow range of aspect ratios, a discotic smectic (DS) phase and also a plastic solid (PS). At high packing fractions and $\kappa^{\mathrm{HP}} \gtrsim 5$ the stable 


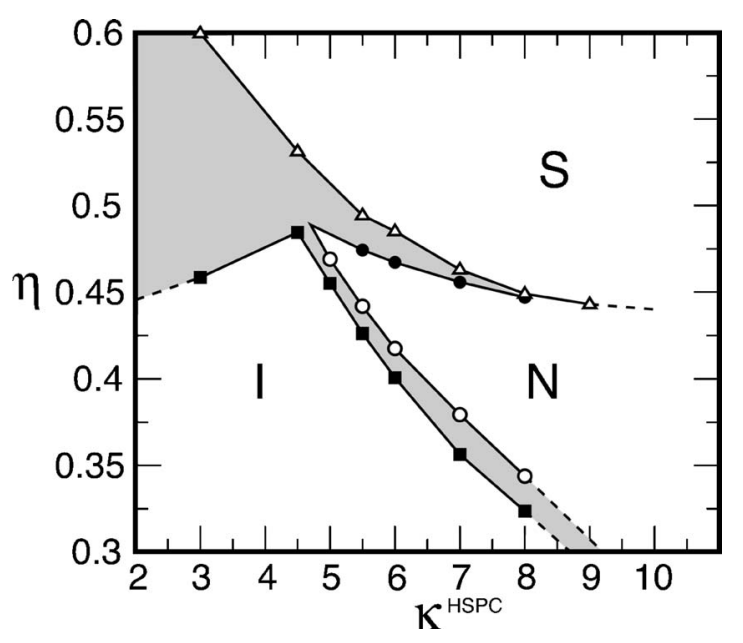

FIG. 1. Phase diagram of a pure system of hard spherocylinders in the packing fraction $(\eta)$-aspect ratio $\left(\kappa^{\mathrm{HSPC}}\right)$ plane, as obtained from the EOT approach. Labels are $N$, nematic; $S$, smectic; and $I$, isotropic.

phase is an orientationally ordered solid (OS). The prediction of a DS phase has been confirmed by simulations of this particular model. ${ }^{12}$ The phase diagram for HSPC obtained with the EOT has not been analyzed in such great detail (see Fig. 1). It contains an $I N$ transition ending at some value of $\kappa$ which is very close to that predicted by the FMT approach for HP particles. The NS transition shows a negative slope, compared with the positive slope obtained in the FMT (at least in the range $5<\kappa<10$ ), and it is of first order up to some value of $\kappa$, above which it becomes second order. By contrast, the NS transition in the FMT approach is always of second order. Since neither the crystalline nor the plasticsolid phases have been explored in the EOT model (mainly due to the fact that this model is not expected, by construction, to provide good results for phases with full spatial order) the comparison of the phase diagrams cannot be extended beyond the liquid-crystalline phases. This is not a limitation for our present purposes, since we would like to

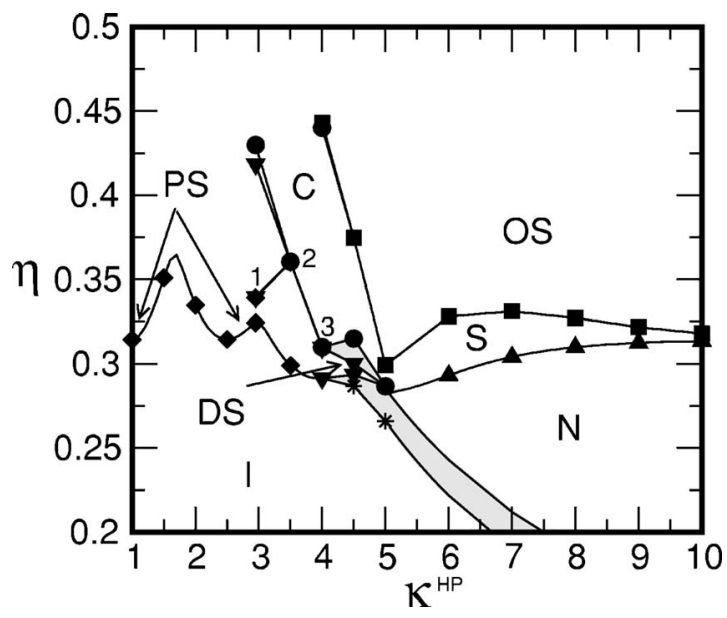

FIG. 2. Phase diagram in the packing fraction $(\eta)$-aspect ratio $(\kappa)$ for a pure component system of hard parallelepipeds, as obtained from the FMT approach. Labels are $N$, nematic; $S$, smectic; OS, orientationally ordered solid; $C$, columnar; PS, plastic solid; and DS, discotic smectic. The symbols indicate the points corresponding to calculated phase coexistence. The numbers indicate that the phase transitions are of first order but that the scale is too small to be seen by the eye.

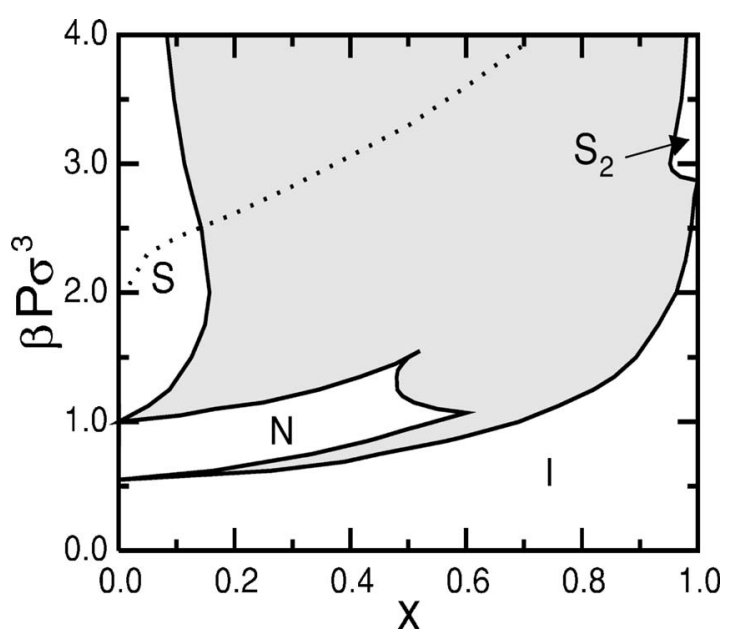

FIG. 3. Phase diagram in the pressure $P$-composition $x$ plane for a mixture of HSPC with the same diameter $\sigma$ and aspect ratios $\kappa_{1}=4.5$ and $\kappa_{2}=8.0$, as obtained from the EOT approach. Reduced units are used for length $(\sigma)$ and energy ( $k T$, the thermal energy). The continuous lines indicate first-order phase transitions. The shaded regions are the two-phase regions of phase coexistence. The regions of stability are labeled by $S$ (standard smectic formed by layers identical in composition), $N$ (nematic), $I$ (isotropic), and $S_{2}$ (microsegregated smectic phase with long particles located in the interlayer space). The dotted line is the spinodal line corresponding to the instability of the nematic phase with respect to columnar-type fluctuations.

focus our investigation on these phases. However, simulations on HSPC indicate that at high packing fractions there appears a solid phase with full order, which becomes a plastic solid when the aspect ratio is low. A more elaborate theory (such as that due to Somoza and Tarazona ${ }^{13}$ ) should be used to address this question.

\section{B. Phase diagrams for mixtures}

We have first investigated the phase behavior of a mixture of particles with $\left(\kappa_{1}, \kappa_{2}\right)=(4.5,8.0)$ and $\sigma_{2} / \sigma_{1}=1$. The results are contained in Figs. 3-6. Throughout this section phase diagrams will be presented in the pressure

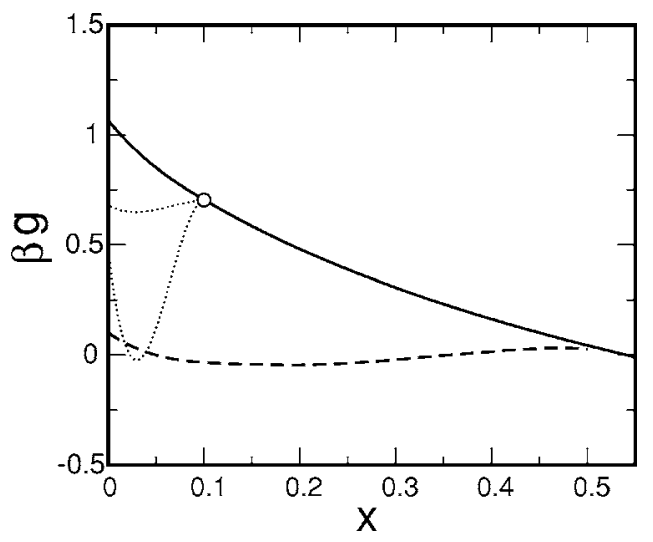

FIG. 4. Gibbs free energy per unit volume and unit thermal energy $\beta g$ as a function of composition $x$ for the nematic phase (continuous line) and the smectic phase (dashed line) for a mixture of HSPC with aspect ratios $\left(L_{1}\right.$ $+\sigma) / \sigma=4.5$ and $\left(L_{2}+\sigma\right) / \sigma=8$.0. A linear term $34.35-12.52 x$ has been subtracted so as to better see the curvature of the curves. The circle is the bifurcation point where the nematic phase becomes metastable with respect to columnar-type fluctuations. The dotted lines are two possible free-energy branches for the columnar phase that could result from a full minimization of the free-energy functional. 


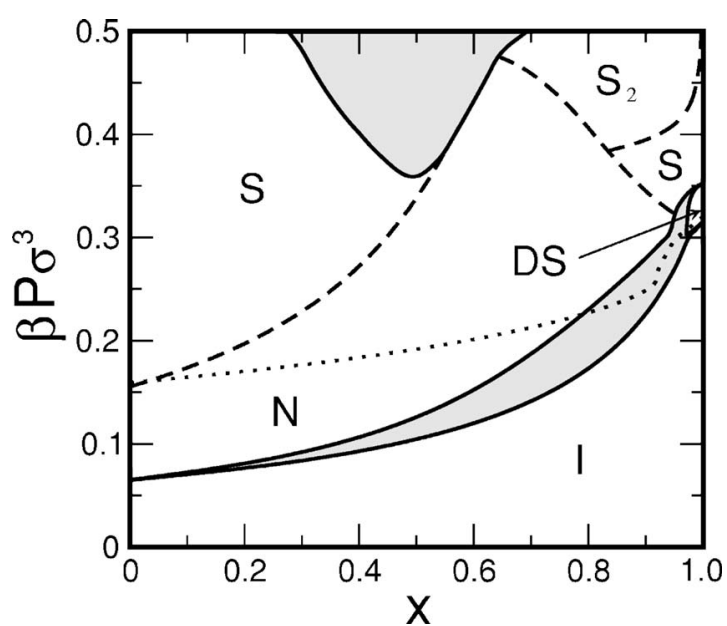

FIG. 5. Phase diagram of the HP mixture in the FMT approach, represented in the pressure-composition plane (reduced units are used for the pressure). All particles have the same cross section $\sigma^{2}$, and its aspect ratios are $\kappa_{1}$ $=4.5$ and $\kappa_{2}=8$. The continuous lines indicate the first-order phase transitions. The shaded areas are the regions of two-phase coexistence. The dashed lines indicate the continuous transitions between the nematic $(N)$ and smectic $(S)$ phases, or between different $S$ phases. The dotted line is the spinodal line signaling instability of the $N$ phase against spatial fluctuations (abrupt change in slope is associated with rapid variation of nematic order parameter). $S$ phase is a standard smectic phase. $S_{2}$ phase consists of alternating layers of different composition. DS is a discotic smectic phase.

$p$-composition $x$ plane (by convention, we take the composition of the mixture to be given by the variable $x \equiv x_{1}$, where species 1 is chosen to correspond to the shortest particles). Figure 3 shows the results as obtained from the Onsager theory (this phase diagram has already been presented in Ref. 4). The most representative feature of this phase diagram is the strong segregation of the $S$ phase, which largely preempts the $I-N$ transition. Two $S$ phases appear: the standard $S$ phase, with identical smectic layers, and the $S_{2}$ phase, where layers of different compositions alternate; this is a microsegregated phase. This phase is characterized by the two variational parameters $\left\{\lambda_{s}\right\}$ having different signs, which implies the density distributions of the two species being shifted one with respect to the other by half a smectic period. Below a reduced pressure $\beta P \sigma^{3}=2.9$ there is direct coexistence be-

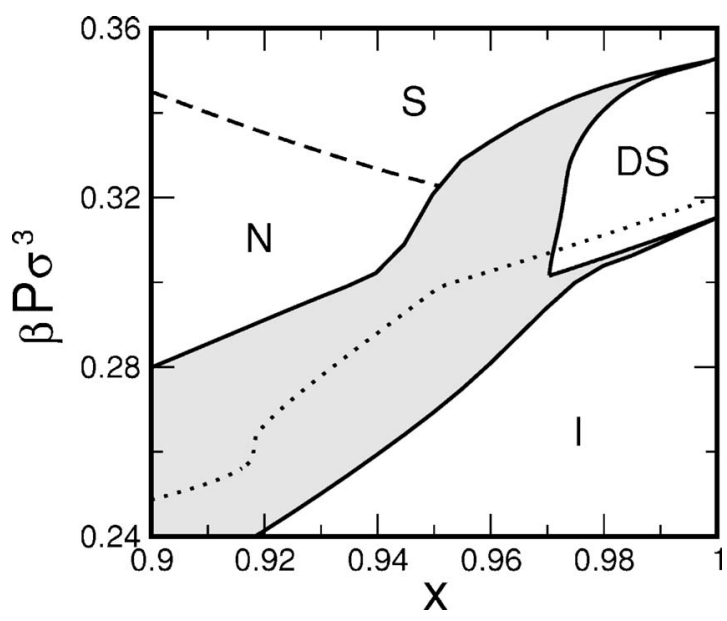

FIG. 6. A zoom of a particular region of the phase diagram represented in Fig. 5 around the DS phase. For a key to lines and labels see caption of Fig. 5. tween the $S$ and $I$ phases, which ends in a $S-N-I$ triple point. Above this pressure the $S$ phase coexists with the $S_{2}$ phase. The Zwanzig model for a comparable mixture is shown in Figs. 4 and 5. Note that the pressure scale is very much reduced with respect to that from the Onsager theory, so that a good reference to compare the two phase diagrams is the location of the $I-N$ transition, which is qualitatively similar to the previous case. In the Zwanzig model $S$ segregation appears at a much higher pressure, and does not preempt the $I-N$ transition. There is no direct $S-I$ transition, which is superseded by continuous $S-N$ transitions, and at high pressure there appears a first-order transition between the standard $S$ phase and the two-layer smectic phase $S_{2}$, the latter appearing when the short-particle component is more abundant; this is in agreement with the predictions based on the Onsager model. However, an important difference is that the $S$ and $S_{2}$ phases undergo continuous phase transitions to the $N$ phase, a feature that is absent in the Onsager model. This is to be expected since in the one-component HP fluid the $N-S$ transition is always of second order. Also, in a model with restricted orientations the $S$ phase is largely destabilized with respect to the $N$ phase, so that the region where the $N$ phase is stable is considerably larger in the Zwanzig model than predicted by the EOT. In addition, the FMT approach predicts also a continuous transition between the $S$ and $S_{2}$ phases in the region where most of the particles correspond to the shorter component. This feature is absent in the EOT approximation.

In order to understand the structure of the $S$ phases in more detail, it is interesting to examine the density and order-parameter profiles along one smectic period $d$ (some of these profiles were shown in Ref. 4 for the EOT model, so here we will only show the profiles corresponding to the FMT approach). This is done in Figs. 7-9. In Fig. 7 the profiles at a state point with reduced pressure $\beta P \sigma^{3}=0.5$ on the $S-S_{2}$ coexistence line are shown. As can be seen the $S$ phase [Fig. 7(a)] is composed of identical layers, with maxima in the density distribution of both species coinciding at the center of each layer. An interesting point to mention is that the position of the short particles is delocalized over the length of the long particles as can be seen from Fig. 7(a). By contrast, in the $S_{2}$ phase, these maxima are shifted by half a period (an amount equal to $d / 2$ ), so that the layers with different compositions alternate. Figure 8 shows profiles at some particular state point on the $S$ - $S_{2}$ second-order transition line. In this point most of the particles in the system are short, and the few long particles present are evenly distributed among the layers and the interstitials. This corresponds to the second-Fourier coefficient $\alpha_{21}^{(1)}$ of the density distribution of the long particles becoming zero at this state point. As $x$ is reduced long particles tend to predominantly populate the insterstitials, defining a bilayer structure. On the contrary, as $x$ is increased, the long particles tend to arrange into the layers formed by the particles of the other component. This behavior is at variance with the predictions of the EOT approach, which imply that long particles added to a smectic phase made up of the short particles tend to populate the interstitials, regardless of their concentration. In Fig. 8 the density profiles of each species along a direction parallel to 


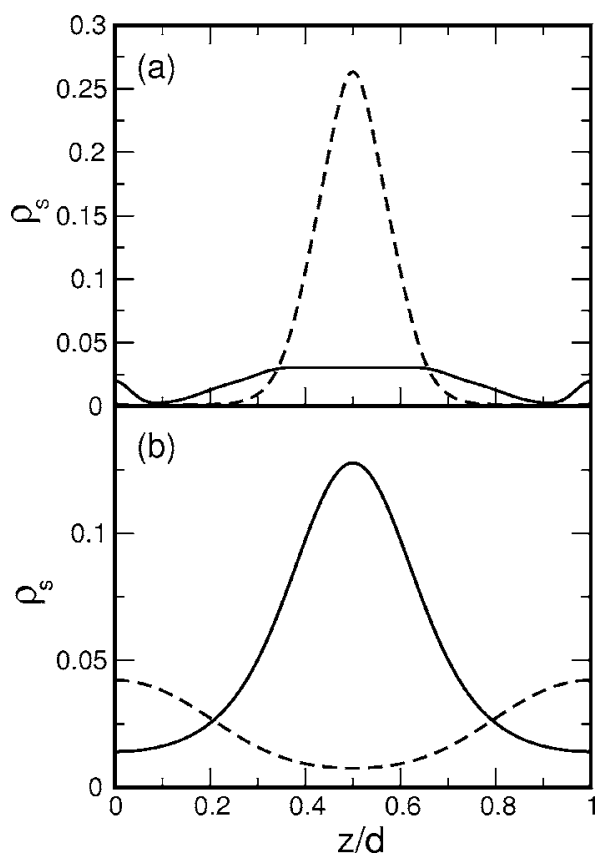

FIG. 7. Density profiles of short (solid line) and long (dashed line) particles of coexisting (a) $S$ and (b) $S_{2}$ phases at pressure $\beta P \sigma^{3}=0.5$. The values of coexistence parameters are $x=0.276, \eta=0.4768$, and $d / L_{2}=1.1642$ for $S$ phase, and $x=0.693, \eta=0.4185$, and $d / L_{1}=1.1498$ for $S_{2}$ phase.

the director are also plotted with dotted lines. These profiles indicate that particles in the layers are mostly directed along the layer normal, whereas short particles tend to align inplane in the interstitials, and a small amount of long particles also adopt this orientation. As can readily be seen, the orderparameter profile also supports this conclusion.

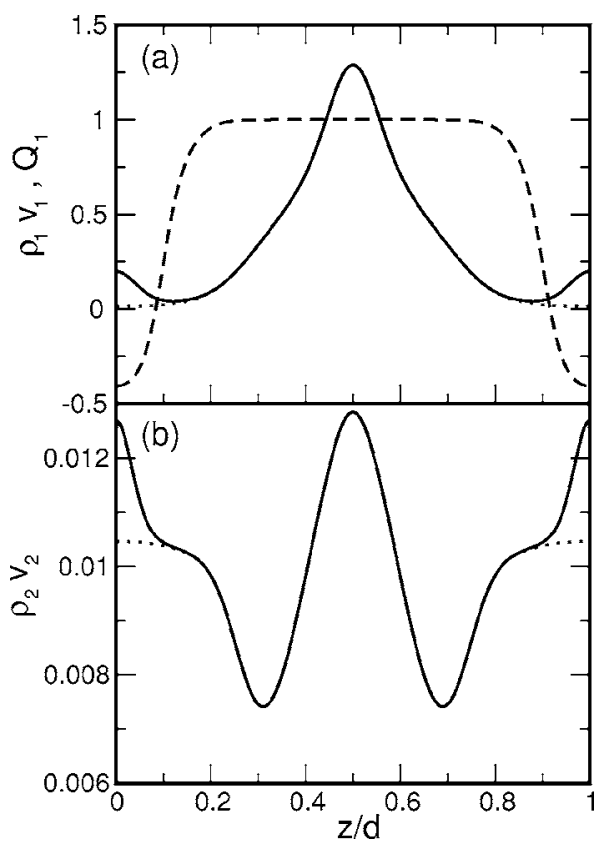

FIG. 8. Density (solid line) and order-parameter (dashed line) profiles of the species with (a) short and (b) long particles. Densities are scaled with the corresponding particle volume $v_{s}$. Density profiles of each species along a direction parallel to the director are plotted with dotted lines. This smectic phase corresponds to a state point in the phase diagram with $\beta P \sigma^{3}=0.442$ and $x=0.9858$. The smectic period in units of the small species length is $d / L_{1}=1.3717$, and the mean total packing fraction $\eta=0.3934$.

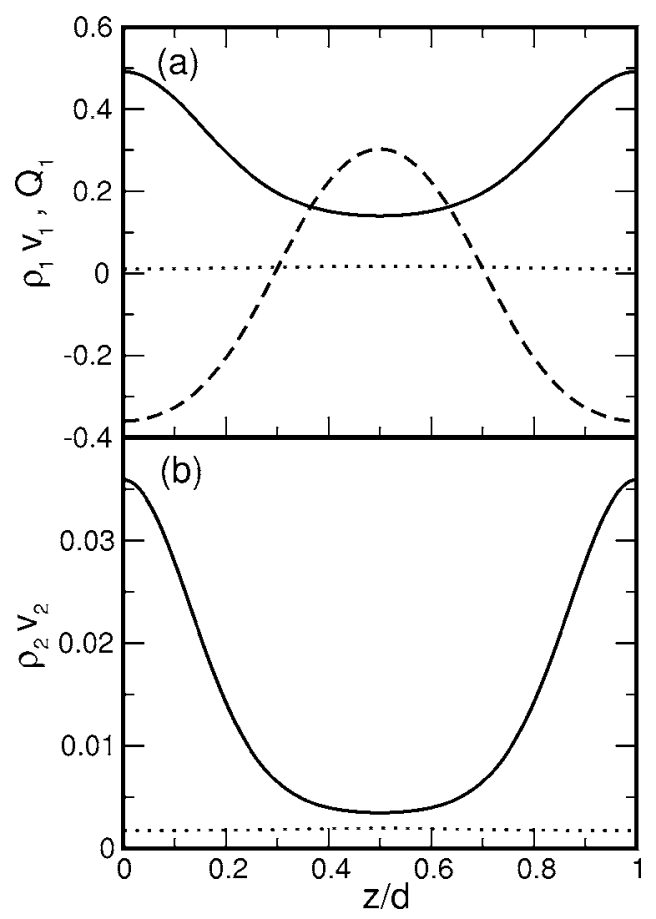

FIG. 9. Density (solid line) and order-parameter (dashed line) profiles corresponding to the species with (a) short and (b) long particles for the discotic smectic phase. Densities are scaled with the corresponding particle volume $\epsilon_{s}$. The projection of the density distribution of each species along the direction of the director is plotted using dotted lines (note that these distributions have maxima at $z / d=0.5)$. The discotic-smectic phase corresponds to the $I$ - $N$-DS triple point with $\beta P \sigma^{3}=0.302$ and $x=0.9715$. The smectic period in units of the cross-section length is $d / \sigma=1.2850$, and the mean total packing fraction $\eta=0.2915$.

An interesting feature of the phase diagram in Fig. 5 is that, for a large concentration of short particles, there is a small region where a discotic-smectic (DS) phase is stable (see Fig. 6, which is an enlargement of Fig. 5 in the region where the DS phase appears). The DS phase was found in a one-component system of HP (Ref. 11) (see Fig. 2, which indicates that for a one-component system with $\kappa=4.5$ there appears a DS phase at packing fractions $\eta \approx 0.3$ ) and confirmed by simulation. ${ }^{12}$ The structure of the DS phase can be understood by examining Fig. 9. We see that the phase consists of a succession of identical layers but, in contrast with the usual smectic phases, particles in the layers are almost exclusively oriented in plane, with nematic order parameter large and negative. The interstitials contain a considerable amount of particles, slightly oriented along the layer normal. The long-particle component tends to follow the majority component and populate the layers. The present result for the mixture indicates that as soon as a small amount of long rods is added to the pure fluid, the DS phase becomes very rapidly unstable.

As an example of another mixture showing strong smectic segregation, but with one species composed on nonmesogenic particles, we discuss the cases $\kappa_{1}=1, \kappa_{2}=8$, and $\sigma_{2} / \sigma_{1}=1$, i.e., mixtures of HSPC with hard spheres or HP with hard cubes (see Figs. 10 and 11). Here again the two models give the same phase behavior: at high volume fractions of the nonmesogenic component we find an isotropic phase, and a relatively small addition of this component to a 


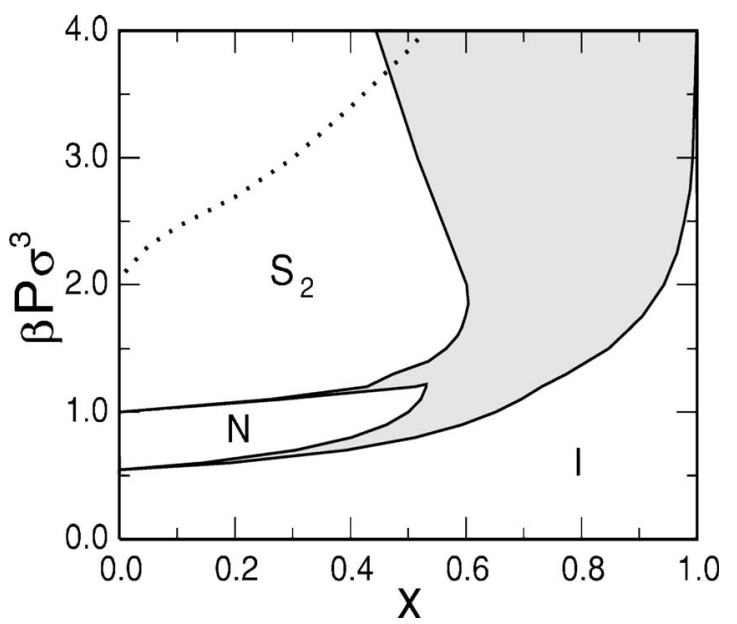

FIG. 10. Phase diagram in the pressure $P$-composition $x$ plane for a mixture of HSPC with the same diameter $\sigma$ and aspect ratios $\kappa_{1}=6.0$ and $\kappa_{2}=8.0$, as obtained from the EOT approach. See caption of Fig. 1 for key to labels and lines.

fluid composed mainly of long particles destabilizes both liquid-crystalline phases. Again the $N-S$ phase is of first order in the Onsager model but of second order in the Zwanzig theory. Apart from that, the two phase diagrams are qualitatively similar. The structure of the smectic phase is interesting, as it is again a clear example of a microsegregated phase: layers rich in long particles alternate with layers made up of (almost exclusively) hard spheres (or hard cubes, as the case may be). Figures 12 and 13 containing density profiles, show this feature for the Zwanzig model (in the case of the EOT the segregation into well-defined layers is even more pronounced $^{4}$ ). Both models predict that the smectic phase is destabilized with respect to the nematic phase as the nonmesogenic component is added ${ }^{14}$ (the slope of the $N-S_{2}$ phase boundary in the pressure-composition plane is positive).

As a final mixture we have studied, using only the Zwanzig approximation, the case where the parallelepipeds and the cubes have the same volumes but different breadths, i.e., different cross sections. The phase diagram corresponding to the cases $L_{1} / \sigma_{1}=8, L_{2} / \sigma_{2}=1, \sigma_{2} / \sigma_{1}=2$ is shown in

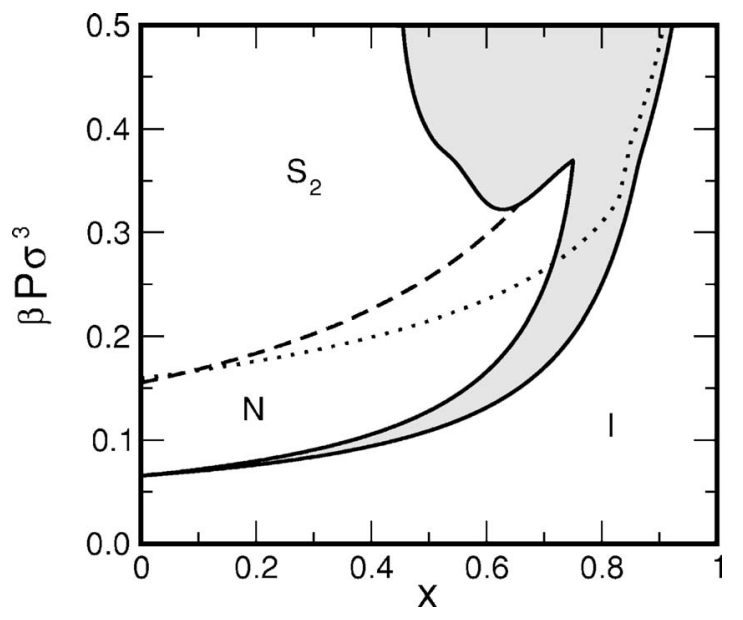

FIG. 11. Phase diagram of a binary mixture of hard parallelepipeds $\left(\kappa_{1}\right.$ $=1)$ and cubes $\left(\kappa_{2}=8\right)$ as obtained from the FMT approach. See caption of Fig. 5 for key to labels and lines.

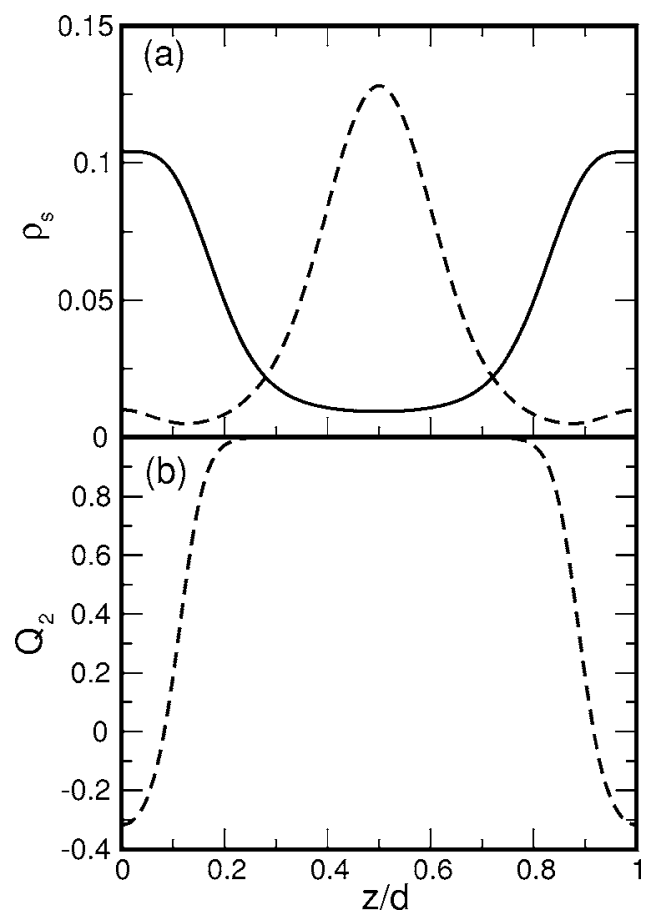

FIG. 12. (a) Density profiles of cubes (solid line) and rods (dashed line) for the coexisting $S_{2}$ phase $\left(\left(\beta P \sigma^{3}=0.37, x=0.543, \quad \eta=0.3579\right.\right.$, and $d / L_{2}$ $=1.6422$ ) at the $I-N-S_{2}$ triple point shown in Fig. 11. (b) Order-parameter profile of rods for the same thermodynamic state.

Fig. 14. Due to the different cross-sectional areas of the particles the smectic phases strongly segregate at high pressures. The $I N$ segregation region is also greatly enhanced. A remarkable feature of the phase diagram is the existence of demixing in the region where the $S_{2}$ phase is stable at low compositions; this phase transition ends in a lower critical

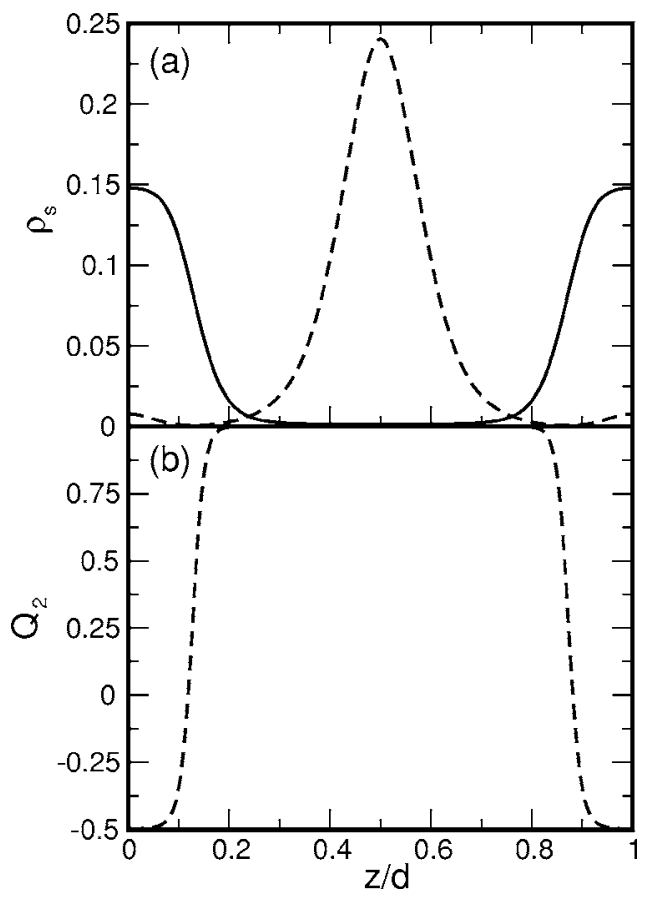

FIG. 13. (a) Density profiles of cubes (solid line) and rods (dashed line) of the $S_{2}$ phase which coexists with the $I$ phase at $\beta P \sigma^{3}=0.5, x=0.455, \eta$ $=0.4414$, and $d / L_{2}=1.5139$ (see Fig. 11). (b) Order-parameter profile of rods for the same thermodynamic state. 


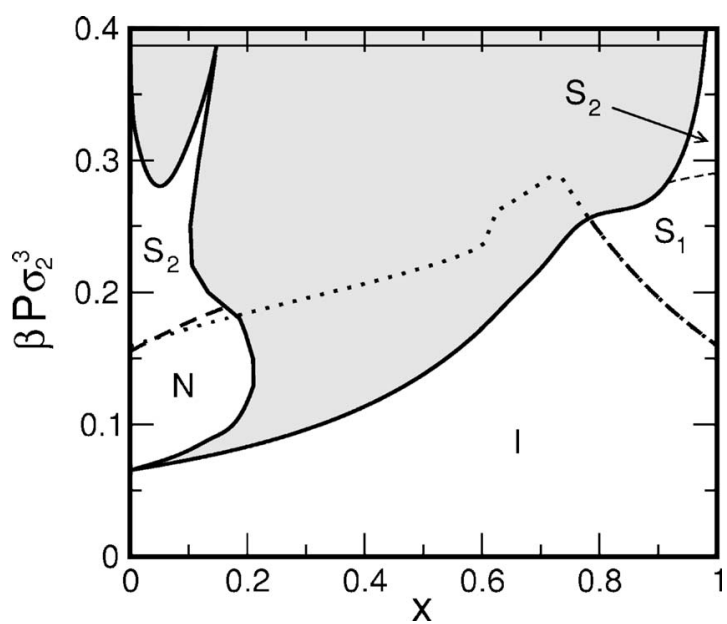

FIG. 14. Phase diagram of a binary mixture of cubes, $\kappa_{1}=1$, and rods, $\kappa_{2}$ $=8$, with $\sigma_{1} / \sigma_{2}=2$ (i.e., with equal particle volumes), as obtained from the FMT approach. The pressure at which the three smectic phases coexist at a triple point is indicated by a horizontal line. See caption of Fig. 5 for key to labels and lines.

point and, at some higher pressure, transforms into a triple point where the two bilayer smectic phases with moderate content of cubes coexist with a third one which is mainly formed by cubes. The density profiles in Figs. 15 and 16 are useful to understand the structure of these phases. In Fig. 15 the density distribution of the first two $S_{2}$ phases is shown. Figure 15(a) shows that there is a very strong microphase separation between the two components which therefore arrange in layers of almost pure composition. The origin of this isostructural phase separation into two bilayer structures can

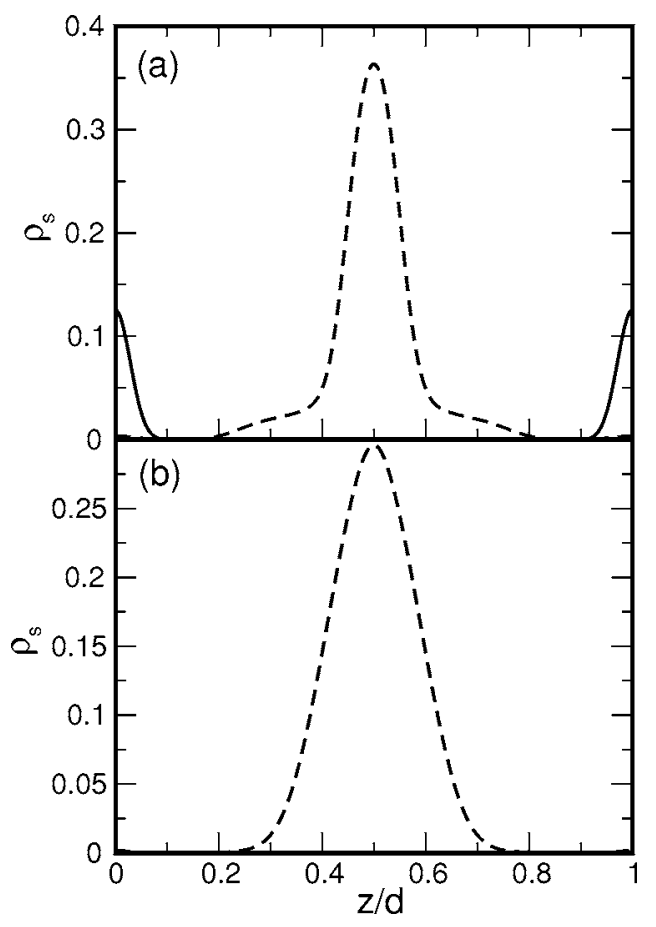

FIG. 15. Density profiles for two of the $S_{2}$ phases that coexist at the triple point with $\beta P \sigma_{2}^{3} \approx 0.388$ (see Fig. 14). (a) Density profiles of cubes (solid line) and rods (dashed line) corresponding to the coexisting $S_{2}$ phase with $x \approx 0.148\left(\eta \approx 0.4816, d / L_{1} \approx 1.4384\right)$. (b) Density profile for the coexisting $S_{2}$ phase with $x_{1} \approx 0.0007\left(\eta \approx 0.4902, d / L_{1} \approx 1.1934\right)$.

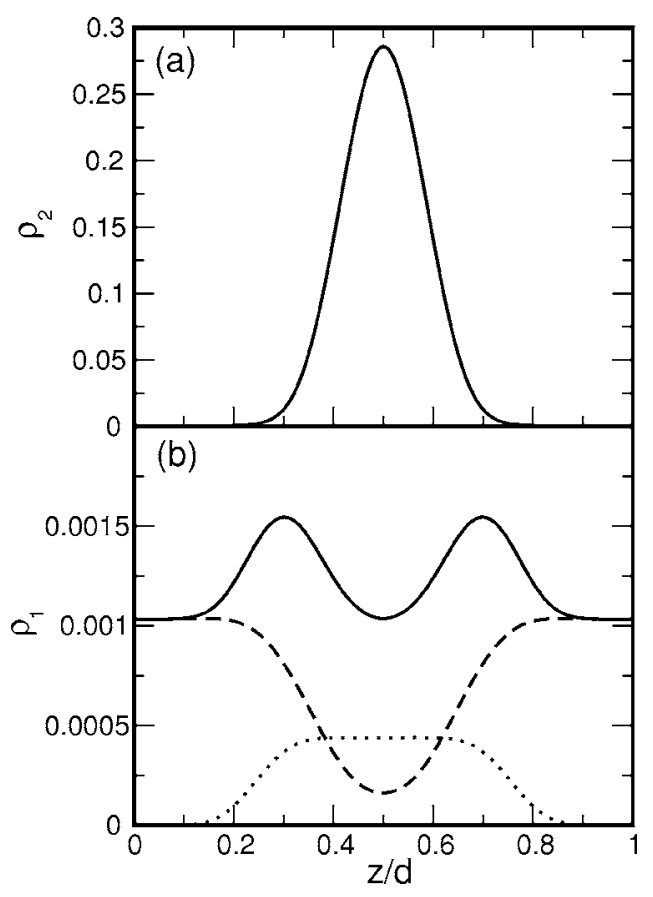

FIG. 16. (a) Density profile of cubes corresponding to the coexisting $S_{2}$ phase at the triple point of Fig. 14 with composition $x \approx 0.98(\eta \approx 0.4860$, $d / L_{2} \approx 1.1839$ ). (b) Density profile of rods at the same thermodynamic conditions; the dotted line represents the density profile corresponding to the rods that lie perpendicular to the director, whereas the dashed line refers to the particles lying parallel to the director. The total density profile is represented by the solid line.

be understood in terms of the so-called depletion effect, which has an entropic origin: the cubes added to a pure mixture of rods in a smectic configuration arrange themselves in the interstitials between the smectic layers, and this arrangement creates an effective attraction between the layers, giving rise to a first-order "condensation" phase transition.

The structure of the bilayer smectic phase at high pressure and with a high content of cubes is also very interesting as it illustrates another effect of the depletion interactions; this is shown in Fig. 16. The thermodynamic state corresponds to the phase that coexists at the triple point, with $x$ $\approx 0.98$. From Fig. 16(a) we can see that the cubes arrange themselves into well-defined layers; however, the rods adopt a very different configuration since they do not occupy the instertitials between layers in a uniform manner: an excess density of rods develops right at the edges of the main distribution of cubes. This is represented in Fig. 16(b) by the solid line, which in turn has been split into parallel and perpendicular components to the director (layer normal) to show that the rods in the excess regions are oriented approximately with equal probability with respect to the three mutual perpendicular directions. We can conclude that the cubes act as a soft wall against which rods are piledup as if adsorbed.

\section{Spinodal lines for columnar order}

The discussion thus far has been focused on the phase diagrams containing $I, N$, and $S$ phases. As discussed in the Introduction, there are strong arguments that suggest that the columnar phase might play a role in the phase stability of the mixtures of hard rods. Using the methodology outlined in 
Sec. II C, we have calculated the spinodal line where the nematic phase becomes unstable with respect to fluctuations involving local spatial order. As implicit in the presentation of the method, our approach does restrict the search for instability to any particular structure; usually, however, the first instability (at the lowest mean density) is found for fluctuations against columnar-type fluctuations (this is signaled by a wave vector with nonvanishing $x$ or $y$ component), except in a few cases to be discussed below.

The spinodal line is represented in the phase diagram of Fig. 3, corresponding to the mixture with $\kappa_{1}=4.5, \kappa_{2}=8$, and $\sigma_{2} / \sigma_{1}=1$, as analyzed in the context of the EOT approach. As can be seen from the figure, the instability occurs at relatively high pressure, which means that there is a relatively wide region of stability for the smectic phase and consequently, the predictions on smectic demixing in mixtures of HSPC are plausible. However, our results indicate that the presence of smectic segregation should be taken with some caution, since it could be preempted by direct coexistence between the $C$ and $S_{2}$ phases. Without a full calculation of the free energy of the $C$ phase this question cannot be settled completely. In order to discuss this point, we plot in Fig. 4 the free-energy branches $g(x)$ of the $N$ and $S$ phases for a mixture with fixed pressure $\beta P \sigma^{2}=2.43$. The bifurcation point is indicated by a circle. Starting at the bifurcation point at $x=0.1$ two possible free-energy branches for the $C$ phase have been represented (the $C$-phase branch will exist for values $x \leqslant 0.1$, see Fig. 3). One possibility is that the curve does not cross the smectic-phase branch: in this case the $C$ phase exists as a metastable phase and the $S$ phase is always more stable. The alternative situation is the one corresponding to the other curve, which crosses the smectic branch: now the $C$ phase becomes more stable and coexists with the $S$ phase. This demonstrates that the bifurcation analysis can only provide trends as to the possible stabilization of the phases and that, once a bifurcation point is shown to exist, one cannot rule out any scenario.

The FMT theory on the HP mixture shown in Fig. 5 predicts a spinodal line at low pressure, below the corresponding line associated with smectic ordering (there is a small region, for small values of $x$, where the smectic line is slightly above the columnar line which leaves a small region of smectic stability; see Figs. 5 and 11). Clearly, the rich smectic phase behavior predicted by the theory is completely preempted by columnar ordering. At high composition $x$ the spinodal line ends in the region of DS stability; this means that the DS phase preempts the instability of the $I$ phase against columnar or plastic-solid fluctuations.

Now we come to show the results for the mixture with $\kappa_{1}=1, \kappa_{2}=8$, and $\sigma_{2} / \sigma_{1}=1$. The results from the EOT for the HSPC model are shown in Fig. 11. We can see that the spinodal line shows the same trend: it increases with composition, and is located at relatively high pressure so that a wide region of smectic stability is found. By contrast, the FMT approach (Fig. 12) again predicts a spinodal line against columnar-type fluctuations below the region of smectic stability, save for a small interval in composition at low values of $x$.

Finally, we have also calculated the spinodal line asso- ciated with columnar fluctuations for the mixture with equal particle volumes but different cross sections, $L_{1} / \sigma_{1}=8$, $L_{2} / \sigma_{2}=1$, and $\sigma_{2} / \sigma_{1}=2$. The results are in the same line as in the previous cases: the smectic phases are completely preempted by the instability against columnar fluctuations. Note that the slope of the spinodal line changes sign at $x \approx 0.7$ and then becomes a spinodal line for instability of the $I$ phase $(0.75 \lesssim x \leqslant 1)$ against spatial fluctuations; in this case the divergence of the structure factor occurs at the same density $\rho$, independent of the orientation of the wave vector, $\mathbf{q}$ $=(0,0, q),(0, q, 0)$, or $(q, 0,0)$, which means that the instability could in principle be associated with the appearance of columnar, smectic, or solid order. This result is analogous to the behavior observed in one-component hard cubes ${ }^{11}$ for which it turns out that the smectic, columnar, and solid freeenergy branches start at the same bifurcation point, the solid phase being the most stable phase. Only a complete densityfunctional minimization could elucidate the relative stability between all these phases in the mixture.

\section{CONCLUSIONS}

In this paper we have analyzed the phase behavior of binary mixtures of hard particles having different geometries, with a view to locating the conditions under which spatially ordered phases are formed. Two different theoretical, somehow complementary, approaches have been used: one based on the standard Onsager theory for hard particles, the other being a fundamental-measure theory for hard parallelepipeds in the Zwanzig approximation, i.e., with restricted particle orientations. It is known that the tendency of the two species to macroscopically segregate is enhanced when the aspect ratio of the particles is more dissimilar. For the more extreme cases this creates large demixing regions where smectic phases of different compositions coexist, even preempting part of the isotropic-nematic transition. Even more significant, in some mixtures the smectic phase exhibits microphase segregation, where the species demix at the level of the one-dimensional smectic unit cell. Microphases may undergo transitions to smectic phases with the usual homogeneous layer composition.

We have demonstrated that these mixtures tend to form columnar phases, even when the cross-sectional length of the particles is unequal, regardless of their relative length. The extent to which columnar order is more or less stable than smectic order has been analyzed using bifurcation theory. In general, the bifurcation analysis of the EOT model predicts that columnar order may appear; whether or not this order preempts direct smectic-phase segregation cannot be ascertained with the present methodology. However, it is clear that large regions of smectic stability still persist in the phase diagram. By contrast, the FMT approach in the Zwanzig approximation predicts that smectic order is almost completely suppressed. The difference in behavior predicted can be traced back to the different treatments of orientational fluctuations (in the latter case orientations are restricted to lie along three orthogonal directions, which enhances columnar order since the order parameter is usually very high). The results obtained with the two approaches probably set the 
limits of what should be expected in the real mixtures. In this respect, given the lack of more reliable theoretical treatments that can give more quantitative answers and the tremendous difficulties involved in making theoretical progress, one should rely on computer simulations, an approach that is feasible but unfortunately not pursued so far.

\section{ACKNOWLEDGMENTS}

One of the authors (Y.M.-R.) was supported by a Ramón y Cajal research contract. This work was partially financed by the Ministry of Education and Science of Spain (DGI), under Grant Nos. BFM2003-0180, BFM2001-0224-C02-01, BFM2001-0224-C02-02, and BFM2001-1679-C03-02.

\section{APPENDIX: DIRECT CORRELATION FUNCTION IN THE FMT APPROACH}

In this section we present explicit expressions for the direct correlation function of the mixtures. In the FMT approach we have, in the nematic phase,

$$
\begin{aligned}
-C_{s \mu, t \nu}\left(\mathbf{r}-\mathbf{r}^{\prime}\right) \equiv & \left.\frac{\delta^{2} \beta \mathcal{F}_{\mathrm{ex}}}{\delta \rho_{s \mu}(\mathbf{r}) \delta \rho_{t \nu}\left(\mathbf{r}^{\prime}\right)}\right|_{\rho_{s \mu}(\mathbf{r})=\rho_{s \mu}=\rho x_{s}\left[1+\left(3 \delta_{\mu z}-1\right) Q_{s}\right] / 3}=\frac{\left\langle\omega_{s \mu}^{(0)} * \omega_{t \nu}^{(3)}\right\rangle+\left\langle\boldsymbol{\omega}_{s \mu}^{(1)} * \boldsymbol{\omega}_{t \nu}^{(2)}\right\rangle}{1-\xi_{3}}+\frac{\boldsymbol{\xi}_{2}\left[\left\langle\boldsymbol{\omega}_{s \mu}^{(1)} * \omega_{t \nu}^{(3)}\right\rangle+\boldsymbol{\Omega}_{s \mu, t \nu}^{(2)}\right]}{\left(1-\xi_{3}\right)^{2}} \\
& +\left[\frac{\xi_{1}}{\left(1-\xi_{3}\right)^{2}}+\frac{2 \boldsymbol{\zeta}_{2}}{\left(1-\xi_{3}\right)^{3}}\right]\left\langle\boldsymbol{\omega}_{s \mu}^{(2)} * \omega_{t \nu}^{(3)}\right\rangle+\left[\frac{\xi_{0}}{\left(1-\xi_{3}\right)^{2}}+\frac{2 \xi_{1} \xi_{2}}{\left(1-\xi_{3}\right)^{3}}+\frac{6 \xi_{2 x} \xi_{2 y} \xi_{2 z}}{\left(1-\xi_{3}\right)^{4}}\right] \omega_{s \mu}^{(3)} * \omega_{t \nu}^{(3)} .
\end{aligned}
$$

The symbol * stands for convolution: $f * g=\int d \mathbf{r}^{\prime} f\left(\mathbf{r}^{\prime}\right) g(\mathbf{r}$ $\left.-\mathbf{r}^{\prime}\right)$ and it is introduced as a shorthand notation. All expressions inside the angular brackets are understood to be symmetrized with respect to their indices $s \mu$ and $t \nu$. For example,

$$
\left\langle\omega_{s \mu}^{(0)} * \omega_{t \nu}^{(3)}\right\rangle=\omega_{s \mu}^{(0)} * \omega_{t \nu}^{(3)}+\omega_{t \nu}^{(0)} * \omega_{s \mu}^{(3)} .
$$

Also, all boldface variables are three-dimensional vectors

$$
\boldsymbol{\xi}_{\alpha}=\left(\xi_{\alpha x}, \xi_{\alpha y}, \xi_{\alpha z}\right), \quad \boldsymbol{\omega}_{s \mu}^{(\alpha)}=\left(\omega_{s \mu}^{(\alpha x)}, \omega_{s \mu}^{(\alpha y)}, \omega_{s \mu}^{(\alpha z)}\right),
$$

with $\alpha=1,2$ and the products between any two of them are to be taken as scalar products. The expression for $\boldsymbol{\Omega}_{s \mu, t \nu}^{(2)}$ is

$$
\boldsymbol{\Omega}_{s \mu, t \nu}^{(2)}=\left(\left\langle\omega_{s \mu}^{(2 z)} * \omega_{t \nu}^{(2 y)}\right\rangle,\left\langle\omega_{s \mu}^{(2 x)} * \omega_{t \nu}^{(2 z)}\right\rangle,\left\langle\omega_{s \mu}^{(2 y)} * \omega_{t \nu}^{(2 z)}\right\rangle\right) .
$$

The expressions for $\xi_{0}$ and $\xi_{3}$ and for the components of $\boldsymbol{\xi}_{\alpha}$ and $\zeta_{2}$ are

$$
\begin{aligned}
& \xi_{0}=\rho, \quad \xi_{3}=\rho \sum_{s} x_{s} v_{s}, \\
& \xi_{1 \beta}=\frac{\rho}{3} \sum_{s} x_{s}\left[2 \sigma_{s}+L_{s}+Q_{s}\left(L_{s}-\sigma_{s}\right)\left(3 \delta_{\beta z}-1\right)\right], \\
& \xi_{2 \beta}=\frac{\rho}{3} \sum_{s} x_{s} v_{s}\left[\frac{2}{\sigma_{s}}+\frac{1}{L_{s}}+Q_{s}\left(\frac{1}{L_{s}}-\frac{1}{\sigma_{s}}\right)\left(3 \delta_{\beta z}-1\right)\right], \\
& \boldsymbol{\zeta}_{2}=\left(\xi_{2 y} \xi_{2 z}, \xi_{2 z} \xi_{2 x}, \xi_{2 x} \xi_{2 y}\right),
\end{aligned}
$$

with $\beta=x, y, z$.

The Fourier transform $\hat{C}_{s \mu, t v}(\mathbf{q})$ of the direct correlation function has the same explicit expression as Eq. (A1), except that the convolutions between different weights $\omega_{s \mu}^{(\alpha)}$ are to be substituted by the products of their corresponding Fourier transforms $\hat{\omega}_{s \mu}^{(\alpha)}$. These are

$$
\begin{aligned}
& \hat{\omega}_{s \mu}^{(0)}(\mathbf{q})=\prod_{\nu=1}^{3}\left[\cos \left(q_{\nu} \sigma_{\mu \nu}^{(s)} / 2\right)\right], \\
& \hat{\omega}_{s \mu}^{(3)}(\mathbf{q})=\prod_{\nu=1}^{3}\left[2 \sin \left(q_{\nu} \sigma_{\mu \nu}^{(s)} / 2\right) / q_{\nu}\right], \\
& \hat{\omega}_{s \mu}^{(1 \alpha)}(\mathbf{q})=\frac{2}{q_{\alpha}} \tan \left(q_{\alpha} \sigma_{\mu \alpha}^{(s)} / 2\right) \hat{\omega}_{s \mu}^{(0)}(\mathbf{q}), \\
& \hat{\omega}_{s \mu}^{(2 \alpha)}(\mathbf{q})=\frac{q_{\alpha}}{2} \cot \left(q_{\alpha} \sigma_{\mu \alpha}^{(s)} / 2\right) \hat{\omega}_{s \mu}^{(3)}(\mathbf{q}),
\end{aligned}
$$

where $\mathbf{q}=\left(q_{1}, q_{2}, q_{3}\right)$ is the weight vector, and $\sigma_{\mu \nu}^{(s)}=\sigma_{s}+\left(L_{s}\right.$ $\left.-\sigma_{s}\right) \delta_{\mu \nu}$.

${ }^{1}$ T. Koda and H. Kimura, J. Phys. Soc. Jpn. 63, 984 (1994).

${ }^{2}$ R. van Roij and B. Mulder, Phys. Rev. E 54, 6430 (1996).

${ }^{3}$ G. Cinacchi, E. Velasco, and L. Mederos, J. Phys.: Condens. Matter 16, S2003 (2004)

${ }^{4}$ G. Cinacchi, L. Mederos, and E. Velasco, J. Chem. Phys. 121, 3854 (2004).

${ }^{5}$ A. Stroobants, Phys. Rev. Lett. 69, 2388 (1992).

${ }^{6}$ A. M. Bohle, R. Holyst, and T. Vilgis, Phys. Rev. Lett. 76, 1396 (1996).

${ }^{7}$ M. A. Bates and D. Frenkel, J. Chem. Phys. 109, 6193 (1998).

${ }^{8}$ J. D. Parsons, Phys. Rev. A 19, 1225 (1979).

${ }^{9}$ S.-D. Lee, J. Chem. Phys. 87, 4972 (1987); J. Chem. Phys. 89, 7036 (1988)

${ }^{10}$ J. A. Cuesta and Y. Martínez-Ratón, Phys. Rev. Lett. 78, 3691 (1997); J. Chem. Phys. 107, 6379 (1997).

${ }^{11}$ Y. Martínez-Ratón, Phys. Rev. E 69, 061712 (2004).

${ }^{12}$ A. Casey and P. Harrowell, J. Chem. Phys. 103, 6143 (1995).

${ }^{13}$ A. M. Somoza and P. Tarazona, Phys. Rev. Lett. 61, 2566 (1988); Phys. Rev. A 41, 965 (1990).

${ }^{14}$ Some predictions by Dogic et al. (Ref. 15) based on Onsager theory indicate that, for parallel hard rods, as their aspect ratio is increased beyond $\kappa_{2} \simeq 10$, the smectic phase should stabilize at the expense of the nematic phase. This effect is related to a more favorable packing of the particles and therefore has an entropic origin (depletion interaction).

${ }^{15}$ Z. Dogic, D. Frenkel, and S. Fraden, Phys. Rev. E 62, 3925 (2000). 